Cite this: DOI: 10.1039/c9tb00593e
Check for updates

\section{Photocrosslinked acemannan-based 3D matrices for in vitro cell culture $\dagger$}

\author{
Simone S. Silva, (D) *ab Diana Soares da Costa (D) ab and Rui L. Reis (D) abc
}

\begin{abstract}
Current biomedical research has highlighted the potential use of medicinal plant derivatives as advanced suitable biomaterials due to their biological properties. Acemannan (ACE) is the main polysaccharide of Aloe vera (AV) leaves with many reported biological properties, including immunomodulatory activity, antibacterial action, wound healing induction, and cytocompatibility. These properties suggest that ACE is an appealing material for the development of 3D structures for biomedical purposes. In this study, ACE was modified by a methacrylation reaction using methacrylic anhydride. Further 3D sponges were developed by processing modified ACE by photocrosslinking using UV irradiation and the freeze-drying technique. Both FTIR and ${ }^{1} \mathrm{H}$ NMR spectroscopy confirmed the effectiveness of the functionalization. SEM and $\mu$-CT revealed that the cross-sections of the methacrylated acemannan (MACE) sponges have open macropores and a narrow pore size distribution within them. We hypothesized that the intrinsic features of ACE could enhance tumor growth, mimicking the biological tumor microenvironment. Therefore, gastric cancer cells (AGS cell line) were cultured in contact with these matrices for up to 14 days to evaluate their cytocompatibility as a processed biomaterial. Results obtained by live-dead assay and MTS showed that cells are viable and metabolically active. Furthermore, confocal laser scanning microscopy analysis showed a homogeneous distribution of cells within the sponge. Therefore, the photocrosslinking on acemmann is proposed as an alternative approach that could significantly improve the processability of acemannan, allowing the production of high added-value structures enabling exploitation of its potential biomedical value.
\end{abstract}

Received 26th March 2019

Accepted 28th May 2019

DOI: $10.1039 / c 9 t b 00593 e$

rsc.li/materials-b

\section{Introduction}

Worldwide, there is a resurgent interest concerning the utilization of bioactive compounds obtained from plant extracts to increase a patients' quality of life. Plant extracts and plant-derived bioactive compounds have been used as sustainable resources to develop new formulations for health care, adsorbents, food supplements, and medical devices because of their presumed safety and potential therapeutic effects. ${ }^{1-4}$ Facing this, current biomedical research has highlighted the potential use of medicinal plant derivatives as advanced suitable biomaterials for cancer management due to their anti-cancer properties. A good example is Aloe vera (AV), an ancient medicinal plant, recognized as a natural resource of bioactive compounds displaying anti-cancer, antioxidant,

\footnotetext{
a $3 B$ 's Research Group, I3Bs - Research Institute on Biomaterials, Biodegradables and Biomimetics, University of Minho, Headquarters of the European Institute of Excellence on Tissue Engineering and Regenerative Medicine, AvePark, Parque de Ciência e Tecnologia, Zona Industrial da Gandra, 4805-017 Barco, Guimarães, Portugal. E-mail: simonesilva@i3bs.uminho.pt

${ }^{b}$ ICVS/3B's - PT Government Associate Laboratory, Braga/Guimarães, Portugal

${ }^{c}$ The Discoveries Centre for Regenerative and Precision Medicine, Headquarters at University of Minho, Avepark, 4805-017 Barco, Guimarães, Portugal

$\dagger$ Electronic supplementary information (ESI) available. See DOI: 10.1039/c9tb00593e
}

anti-bacterial and chemo-preventive properties. ${ }^{5}$ Acemannan, ACE, the main polysaccharide of AV leaves, is a $\beta(1 \rightarrow 4)$-acetylated polymannose, ${ }^{6}$ known for its cytocompatibility, wound healing inducer capability, antibacterial action, and immunomodulator activity. ${ }^{5,7}$ ACE acts as a bioactive molecule, exerting an immunostimulatory effect by activating macrophages, ${ }^{8,9}$ stimulating the expression of vascular endothelial growth factor (VEGF), keratinocyte growth factor-1 (KGF-1) and type I collagen. ${ }^{10}$ Besides that, ACE has been reported to stimulate gingival fibroblast proliferation, periodontal tissue regeneration, ${ }^{11}$ soft tissue regeneration, ${ }^{12}$ cementoblast proliferation, ${ }^{13}$ and bone marrow stromal cell proliferation and differentiation in vitro. ${ }^{14}$ These studies suggest that ACE can be an appealing biomedical material. In most of these investigations, ACE was used in its powder form. Therefore, to our knowledge, no research study has been reported so far, exploring the preparation of ACE-based 3D structures.

We investigate the functionalization of ACE through its reaction with methacrylic anhydride. Furthermore, modified ACE is photocrosslinked in the presence of small amounts of Irgacure 2959 (photoinitiator), and the obtained hydrogels were further freeze-dried to obtain sponges. Photocrosslinking reactions on natural polymers offer considerable advantages such as a rapid curing reaction, low energy consumption, and low-cost compared 
to traditional chemical reactions. ${ }^{15-17}$ Beyond that, photocrosslinking allows 3D hydrogel networks to be produced with controlled shape and size. In our group, natural polymers such as fucoidan, ${ }^{18}$ gellan gum ${ }^{19}$ and $k$-carragenan ${ }^{16}$ were modified already using specific unsaturated functional groups (e.g., glycidyl methacrylate, methacrylic anhydride). These groups undergo free radical polymerization in the presence of a photoinitiator upon ultraviolet (UV) or light irradiation, creating a variety of matrices for different biomedical applications.

3D-based matrices composed of natural polymers, namely silk fibroin, chitosan, collagen, and their blends, ${ }^{20-23}$ can represent a better alternative to conventional $2 \mathrm{D}$ cell culture for understanding cell metabolism and behavior, allowing creation of accurate tumor models. The $3 \mathrm{D}$ in vitro cancer models can mimic a natural microenvironment of tumors more similar to that found in vivo. ${ }^{24}$ As a proof of concept, the in vitro cellular viability within the acemannan-based sponges was evaluated using the AGS gastric-cancer cell line as an assessment of their biocompatibility.

\section{Experimental}

\section{Materials}

Acemannan in powder form (ACE; BiAloe ${ }^{\circledR} M_{\mathrm{w}}$ average $200 \mathrm{kPa}$ ) was a gift from Lorand Laboratories (Texas, USA). Polydimethylsiloxane (PDMS) (Sylgard184) was obtained from Dow Corning. Methacrylic anhydride (MA) and 2-hydroxy-40-(2-hydroxy-ethoxy)-2methyl-propiophenone, 98\% (Irgacure2959) were purchased from Sigma-Aldrich. All the reagents were used as received.

\section{Methods}

Synthesis of methacrylated acemannan. Methacrylated acemannan (MACE) was synthesized by reacting ACE with methacrylic anhydride (MA). Briefly, ACE was dissolved at $2.5 \%(\mathrm{w} / \mathrm{v})$ into deionized water at $50{ }^{\circ} \mathrm{C}$ until the polymer was fully solubilized. To this solution, MA ( $8 \%$ in relation to the solution used) was added and allowed to react for 6 hours at $50{ }^{\circ} \mathrm{C}$. The $\mathrm{pH}$ (8.0) of the reaction was periodically adjusted with 5.0 M NaOH solution in distilled water. MACE solutions were dialysed against distilled water using 12-14 $\mathrm{\kappa}$-CA cut off dialysis tubing (Fisher Scientific, USA) for 3 days at room temperature to remove excess unreacted MA. MACE solutions after the dialysis process were frozen at $-80{ }^{\circ} \mathrm{C}$ and then lyophilized. The obtained powder was purified dispersing it in pure ethanol with successive changes during 48 hours. Then, the purified polymer was frozen at $-80{ }^{\circ} \mathrm{C}$ and lyophilized again. The lyophilisation step was performed at $0.08 \mathrm{mbar}$ and temperature $-77.7{ }^{\circ} \mathrm{C}$. The obtained powder was protected from light and stored at $4{ }^{\circ} \mathrm{C}$, until further use.

Preparation of crosslinked MACE hydrogels. Freeze dried MACE was added to a photoinitiator (PI) solution consisting of 0.25\% (wt/v) 2-hydroxy-1-(4-(hydroxyethoxy)phenyl)-2-methyl-1propanone (Irgacure D2959, CIBA Chemicals), in distilled water at room temperature with stirring until complete dissolution. Chemically crosslinked MACE hydrogels were obtained by pipetting $100 \mu \mathrm{L}$ of the polymer solution into PDMS circular molds
(8 $\mathrm{cm}$ diameter), followed by UV light exposure at $6.9 \mathrm{~mW} \mathrm{~cm} \mathrm{~cm}^{-2}$ (320-480 nm, EXFO OmniCure S2000, Ontario, Canada) for 60 seconds. After exposure, the fabricated hydrogels were placed in distilled water for 24 hours. Then, the hydrogels were frozen at $-80{ }^{\circ} \mathrm{C}$ overnight and freeze-dried.

\section{Characterization}

FT-IR spectra were recorded as $\mathrm{KBr}$ pellets $(1 / 100 \mathrm{mg})$ in the range $4000-400 \mathrm{~cm}^{-1}$ by using a Jasco FT-IR 410 spectrophotometer with a resolution of $4 \mathrm{~cm}^{-1}$. Each spectrum was recorded after 16 scans.

The methacrylation of acemannan was quantified by ${ }^{1} \mathrm{H}$ NMR spectroscopy. The ${ }^{1} \mathrm{H}$ NMR spectra of acemannan and MACE were collected in deuterated water $\left(\mathrm{D}_{2} \mathrm{O}\right) 5 \mathrm{mg} \mathrm{mL}^{-1}$ and $50{ }^{\circ} \mathrm{C}$, being recorded on a Burker Avance III, using the following spectral conditions: $300 \mathrm{~Hz}$ spectra with $90^{\circ}$ impulses, $4 \mathrm{~s}$ acquisition time. Spectra were processed by using Mestre Nova software. The methacrylation degree (DM) was calculated referring to the peaks at $\delta=3.1-4 \mathrm{ppm}(\mathrm{H} 2-6)$ and $\delta=5.7-5.9 \mathrm{ppm}$ (double bound region) as a percentage (\%) of the hydroxyl groups $(-\mathrm{OH})$ substituted with methacrylate groups.

The zeta potentials of the acemanann (ACE) and modified acemannan (MACE) were measured by laser Doppler anemometry using a Malvern Zetasizer 3000 HS (Malvern Instruments, UK). Each sample was dissolved in distilled water at a concentration between $0.5-5 \%(\mathrm{w} / \mathrm{v})$. Each analysis was made at $25{ }^{\circ} \mathrm{C}$ for 60 seconds. Independent triplicate experiments were performed for obtaining statistical significance.

Samples of the freeze-dried hydrogels were observed by a NanoSEM-FEI Nova 200 (FEG/SEM) Scanning Electron Microscope equipped with an EDX: Pegasus X4M instrument. The materials were fixed by mutual conductive adhesive tape on aluminum stubs and covered with gold-palladium using a sputter coater.

The microstructure of the scaffolds was evaluated using a high-resolution $\mu$-CT Skyscan 1072 scanner (Skyscan, Kontich, Belgium) with a resolution of the pixel size of $6.69 \mu \mathrm{m}$ and an integration time of $1.9 \mathrm{~s}$. The X-ray source was set at $71 \mathrm{keV}$, and $140 \mu \mathrm{A}$ and 300 projections were acquired over a rotation range of $180^{\circ}$ with a rotation step of $0.45^{\circ}$. Standardized cone-beam reconstruction software (NRecon v1.4.3, SkyScan) was used to reconstruct data sets. The output format for each sample was 300 serial $1024 \times 1024$ bitmap images. A representative data set of 300 slices was segmented into binary images with a dynamic threshold of 38-255 (grey values) and 120-255, for analysis of scaffolds and ceramic components alone, respectively. The same representative volume of interest (VOI) was analysed for all the samples. These data sets were used for morphometric analysis (CT Analyser, v1.5.1.5, SkyScan) and to build the 3D models (ANT 3D creator, v2.4, SkyScan).

Swelling tests were performed by immersing the MACE sponges in PBS (pH 7.4) for up to $1440 \mathrm{~min}$. The swollen sample weights were measured after removing excess surface water by gently tapping the surface with filter paper. Eqn (1) was used to 
calculate the percentage of water uptake, where $W_{\mathrm{s}}$ is the weight of the swollen sample, and $W_{\mathrm{d}}$ is the weight of the dry sample. Each experiment was repeated three times, and the average value was considered to be the water uptake value.

$$
\text { Water uptake }(\%)=\left(\left(W_{\mathrm{s}}-W_{\mathrm{d}}\right) / W_{\mathrm{d}}\right) \times 100
$$

The stability of MACE sponges $(1 \times 1 \mathrm{~cm})$ was evaluated by immersing them in PBS solution at $37{ }^{\circ} \mathrm{C}$ for 21 days to analyze their stability in an aqueous medium. Samples were collected at 7, 14 and 21 days, washed gently with distilled water, dried at $40{ }^{\circ} \mathrm{C}$ and weighed. Percentage loss in weight at the respective time points was calculated.

\section{Cell seeding and culture on sponges}

Before testing, the samples were sterilized in an ethylene oxide atmosphere. In vitro cell tests were performed using a cell suspension of the AGS gastric-cancer cell line (passage 8), at a concentration of $7.5 \times 10^{5}$ cells per sponge (with an approximate volume of $0.125 \mathrm{~mm}^{3}$ ). Triplicates were made for each sample. The cell-sponge constructs were incubated at $37{ }^{\circ} \mathrm{C}$ in a humidified 95\% air-5\% $\mathrm{CO}_{2}$ atmosphere and maintained for 1 , 7, and 14 days. Cells were cultured in Roswell Park Memorial Institute medium (RPMI 1640, Sigma) supplemented with 10\% foetal bovine serum (FBS Gibco, UK) and 1\% antibiotic/antimycotic (Gibco, UK) and the culture medium was replaced every other day. The medium was removed after 1, 7 and 14 days of culture, and the samples were washed with a PBS solution, and either directly used (live-dead assay, MTS test, and DNA quantification) or fixed with $10 \%$ formalin solution (Sigma, USA) for 30 minutes and used for microscopy analysis.

The viability of the cells was evaluated by the live-dead assay. Briefly, cells were incubated in a mixture of calcein AM $\left(2 \mu \mathrm{g} \mathrm{mL}{ }^{-1}\right)$ and propidium iodide $\left(1 \mu \mathrm{g} \mathrm{mL} \mathrm{m}^{-1}\right)$ in PBS for $10 \mathrm{~min}$ at room temperature. Images of the constructs were obtained using confocal laser scanning microscopy (CLSM, Leica TCS SP8). Cell morphology and spreading of AGS seeded onto MACE sponges was monitored by CLSM. For this purpose, after each culturing period, the cellsponge constructs were incubated with $3 \%$ bovine serum albumin (BSA) for $30 \mathrm{~min}$. The AGS cells were permeabilized with $0.1 \%$ Triton X-100 in PBS for 5 min, and incubated with phalloidin-TRITC for $20 \mathrm{~min}$ at room temperature, followed by washing with PBS and staining with $5 \mu \mathrm{g} \mathrm{mL}{ }^{-1}$ DAPI for $30 \mathrm{~min}$. Fluorescence images from stained constructs were obtained using a CLSM (Leica TCS SP8).

The metabolic activity of the AGS gastric cancer cells seeded on the MACE sponges was evaluated using the CellTiter $96^{\circledR}$ AQueous One Solution Cell Proliferation Assay (MTS, Promega). Briefly, cell-sponge constructs were placed in a mixture containing serum-free DMEM Low glucose (Sigma Aldrich) and MTS reagent at a $5: 1$ ratio and incubated for $3 \mathrm{~h}$ at the end of which the optical density was read (OD $=490 \mathrm{~nm})$.

AGS cell proliferation onto MACE sponges was determined using a fluorimetric double strand DNA quantification kit (PicoGreen, Molecular Probes, Invitrogen, UK). For this purpose, samples collected at 1,7 , and 14 days were washed with PBS and transferred into $1.5 \mathrm{~mL}$ tubes containing $1 \mathrm{~mL}$ of ultra-pure water. AGS cells-sponge constructs were incubated for one hour at $37{ }^{\circ} \mathrm{C}$ in a water-bath and then stored in a $-80{ }^{\circ} \mathrm{C}$ freezer until testing. Before the dsDNA quantification, the constructs were thawed and sonicated for $20 \mathrm{~min}$. The quantification was performed in triplicate according to supplier instructions using a microplate ELISA reader (BioTek, USA).

All quantitative experiments were run in triplicate, and the results are expressed as a mean \pm standard deviation for $n=3$. Statistical analysis of the data was performed using two-way ANOVA with Bonferroni's post-test by using GraphPadPrism version 5.0 for Windows (GraphPad Software, San Diego, http:// www.graphpad.com). Differences between the groups with $p<0.05$ were considered to be statistically significant.

\section{Results and discussion}

ACE has been recently pointed out as an appealing biomedical material for tissue engineering applications due to its favorable biocompatibility and bioactivity. ${ }^{7,14,25}$ Although these studies have shown encouraging results, the processing of ACE as a $3 \mathrm{D}$-based structure is still limited. To fulfill this gap, we propose the modification of ACE with methacrylate pendant groups, yielding photocrosslinkable hydrogels. The functionalization of polysaccharides using methacrylation reactions, followed by photopolymerization, is not a new approach. ${ }^{16,18,26}$ However, their application on ACE to produce 3D hydrogel networks has not been reported yet. The covalent bonds formed during the methacrylation reaction are expected to enhance the stability of the ACE in aqueous media. Scheme 1 illustrates the possible chemical reaction leading to ACE methacrylation and further photocrosslinking using UV irradiation. The scheme proposed is only one hypothesis for the modification position, that can occur in any hydroxyl groups present in the ACE chain. The ${ }^{1} \mathrm{H}$ NMR spectra of ACE showed that the $O$-acetyl groups (HAc) of ACE occur at chemical shift $\sim 1.9 \mathrm{ppm}$, while H2-6 occurs at 3.1-4.0 ppm (Fig. 1A). ${ }^{27} \mathrm{H}$ NMR confirmed the methacrylation by the presence of the peaks: $\delta=1.9-2.0 \mathrm{ppm}$ (overlapping of methyl and $O$-acetyl groups) and $\delta=5.7-5.9$ (corresponds to sugar rings at $\mathrm{H} 2-6$ protons) (Fig. 1A). The calculated methacrylation degree was about 25\%. We do expect that upon ACE modification bioactivity is maintained since its biocompatibility properties are relevant for its employment as building blocks for these hydrogels.

Structural differences between ACE and methacrylated ACE (MACE) were analysed by FTIR analysis (Fig. 1B). On the spectrum of ACE (Fig. 1B), the main characteristic bands can be observed, namely the presence of stretching vibration of $O$-acetyl groups at $1731 \mathrm{~cm}^{-1}$ and $1247 \mathrm{~cm}^{-1}$, phenolic $=\mathrm{CO}$ (corresponds to $1602 \mathrm{~cm}^{-1}$ ), COO- (corresponds to $1435 \mathrm{~cm}^{-1}$ ) and -COC (corresponds to $1036 \mathrm{~cm}^{-1}$ ). ${ }^{27}$ The confirmation of the modification was observed through a small displacement of the mentioned bands to higher wave-numbers (Fig. 1B). In the MACE spectrum (Fig. 1B), a carbon-carbon double bond peak at $1638 \mathrm{~cm}^{-1}$, and a peak related to a carbonyl at $1736 \mathrm{~cm}^{-1}$, related to the methacrylate group, appeared overlapping with other vibrational modes of the ACE, which supports the confirmation of the methacrylation of ACE, previously observed by NMR results. 
(A)
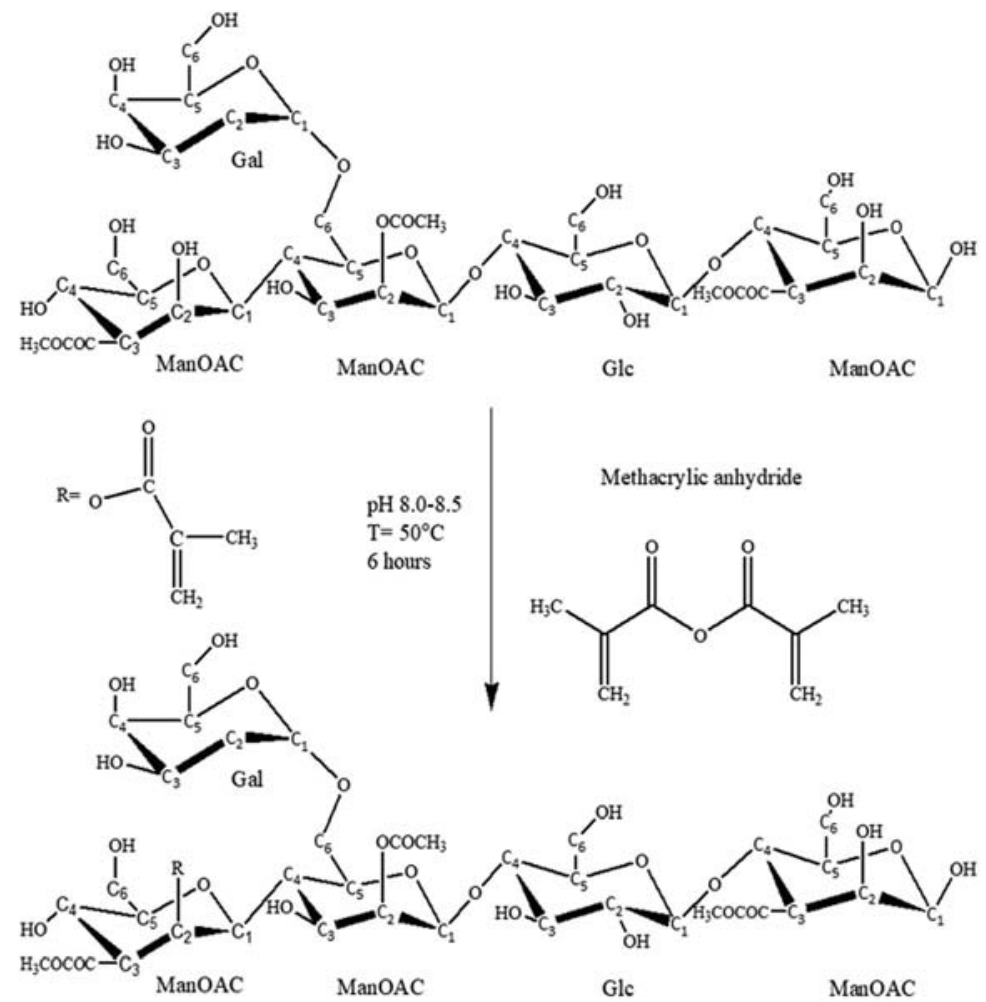

(B)

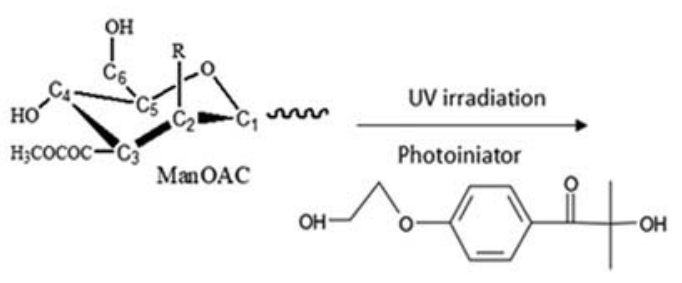

Irgacure D2959

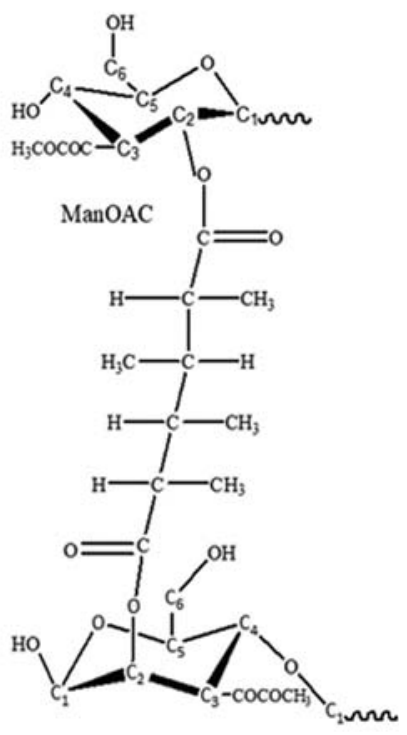

ManOAC

Scheme 1 Scheme of the possible mechanism of acemannan and methacrylated anhydride (A) and further photopolymerization (B).

The introduction of methacrylate groups into the ACE backbone enables the formation of chemically crosslinked gels upon irradiation with UV exposure for 60 seconds (Fig. 1C). ACE hydrogels were obtained using different concentrations of modified ACE, namely 2.5, 5, and $10 \mathrm{wt} \%$. The ACE based hydrogels have a certain opacity (Fig. 1C), and they showed good structural stability without disintegration during manipulation and washing steps. Developed architectures using the highest concentration of modified ACE ( 5 and $10 \mathrm{wt} \%$ ), have a compact structure (data not shown). Therefore, the ACE concentration of $2.5 \mathrm{wt} \%$ was chosen for further characterization.

From the SEM images of the cross-section of the freeze-dried ACE hydrogels, it is possible to observe a typical sponge three dimensional (3D) morphology with open macropores (Fig. 2A). The quantitative analysis of the ACE sponges was investigated by micro-CT. 3D $\mu$-CT images (Fig. 2B and C), obtained from 

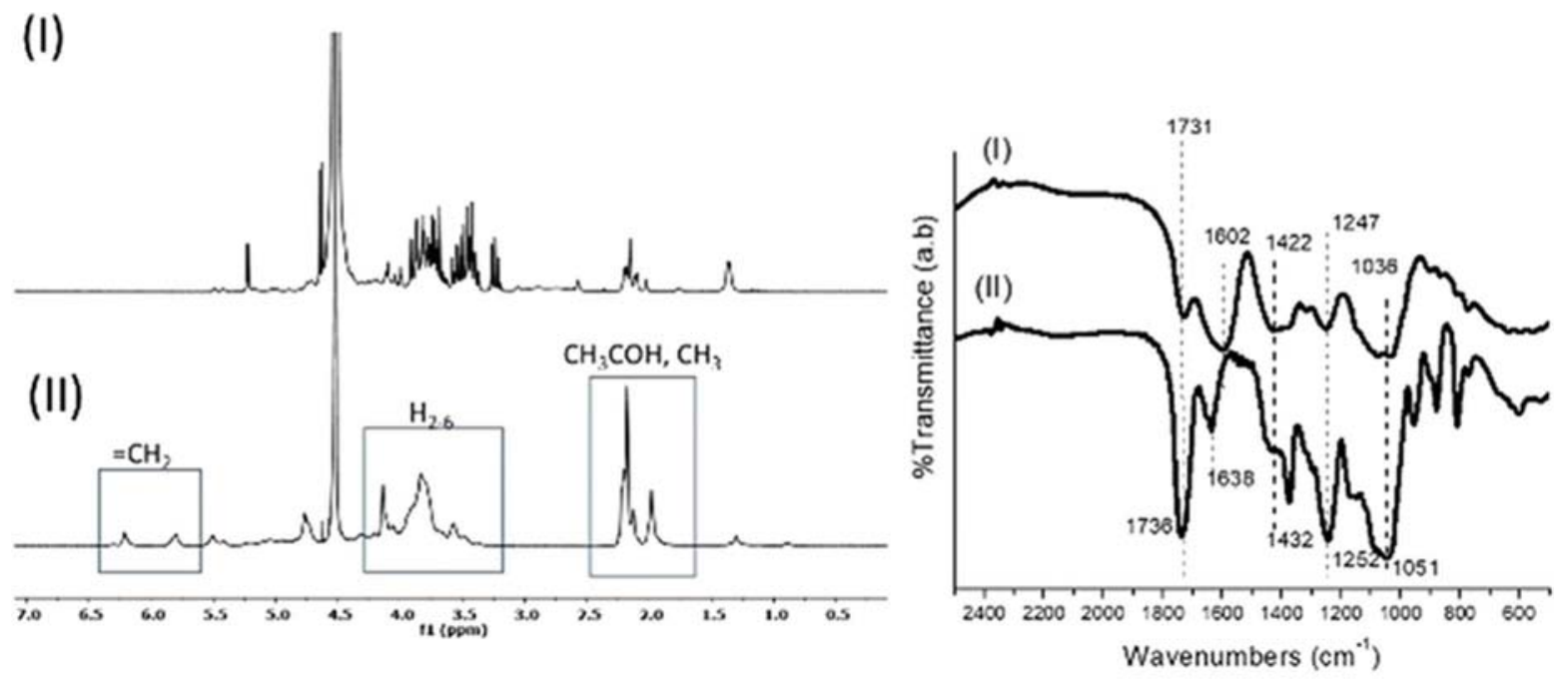

(C)

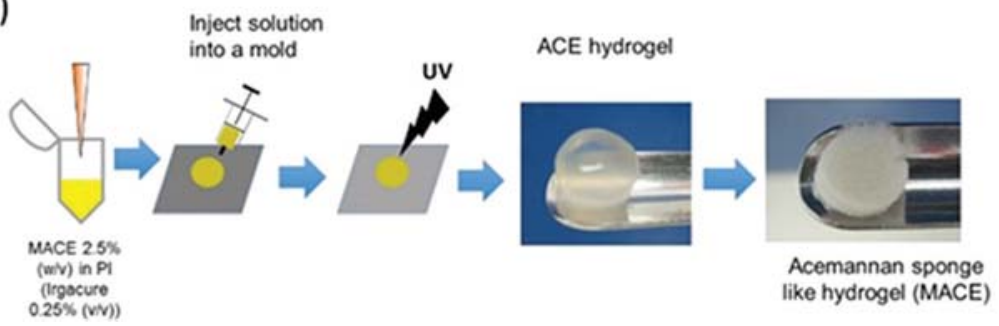

Fig. 1 (A) ${ }^{1} \mathrm{H}$ NMR spectrum of acemannan (I) and methacrylated acemannan (II) performed in $\mathrm{D}_{2} \mathrm{O}$ at $50{ }^{\circ} \mathrm{C}$; (B) FTIR spectra of acemannan (I) and methacrylated acemannan (II); (C) schematic illustration of the preparation of crosslinked MACE matrices.

digital geometry processing from a series of two-dimensional $\mathrm{X}$-ray images demonstrate that the structures present a mean pore size of $109.1 \pm 0.9 \mathrm{~mm}$, and a porosity of $38.1 \pm 0.7 \%$, with interconnected pores of $30.1 \pm 0.9 \%$. The micro-CT morphometric analysis revealed a narrow pore size distribution within the sponges (Fig. 2D).

The zeta potential measurements confirmed the anionic potential of both ACE and MACE, where the values found were $-9.4 \pm 0.4$ and $-3.9 \pm 0.1 \mathrm{mV}$, respectively. This result suggests that the chemical modification performed on ACE yielded a polymer with slightly positively charged surfaces as compared to the unmodified ACE.

\section{Swelling and stability}

The swelling behavior of MACE sponges was assessed by immersing them in PBS solution at $37^{\circ} \mathrm{C}$. The sponges were stable in the PBS and presented an increase in the water uptake up to $240 \mathrm{~min}$, and a slight decrease after that point. MACE sponges offer large free spaces (porous) between the crosslinked networks, increasing their water uptake ability (Fig. 3A). Moreover, the MACE sponges are composed by hydrophobic methacrylate groups in the chain (DM about 25\%), leading to a structure that is stable in an aqueous medium.

To evaluate the stability of the structures in an aqueous medium for a prolonged time, the MACE sponges were immersed in PBS for
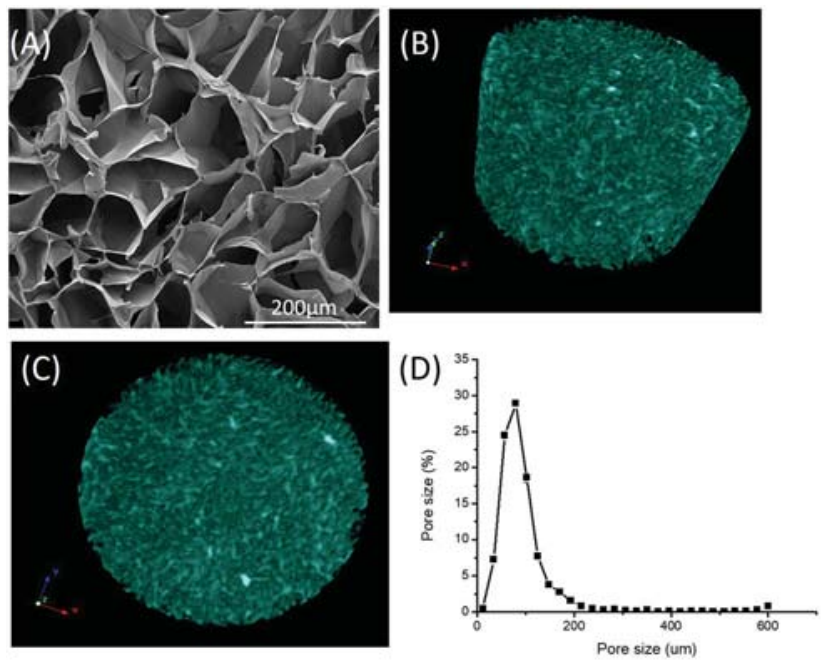

Fig. 2 Cross-section of the MACE sponges (A), 3D reconstruction of the MACE sponges by micro-CT, (B) lateral view and top view (C) and pore size distribution of MACE (D).

up to 21 days. The samples showed a weight loss of about $22 \%$ after a longer immersion time (21 days), confirming the high stability of MACE sponges in an aqueous medium (Fig. 3B). The weight loss observed during the performed stability tests could be related to a 

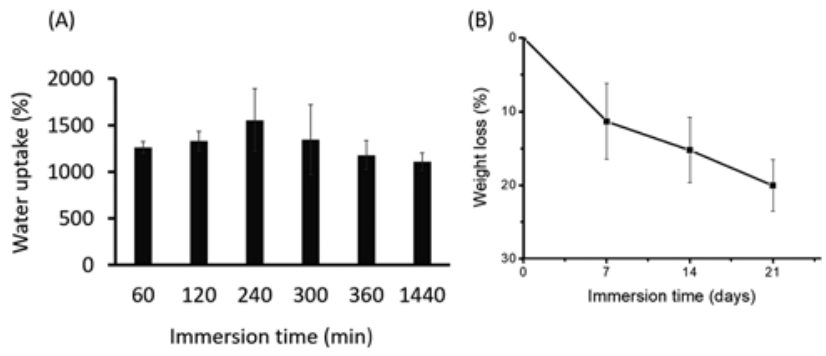

Fig. 3 Water uptake (A) and weight loss (B) of MACE sponges after immersion in PBS under stirring at $37^{\circ} \mathrm{C}$.

fragmentation of the samples, considering the absence of enzymes. Thus, the introduction of hydrophobic methacrylate groups in the ACE chain stabilized the structure of the sponge which resulted in a slower degradation rate.

\section{Biological behaviour}

Previous reports have shown that AGS cells need lower stiffness values in 3D models to maintain cancer stem cell (CSC) growth and therefore, mimic cancer aggressiveness in vivo. ${ }^{26}$ The adhesion and viability of AGS cells on MACE sponges was monitored throughout the experiment by the live-dead assay. Cells remain viable until 14 days both at the surface and inside the sponges (Fig. 4). Calculations of the percentage (\%) of live/dead cells were performed (see Fig. S1, ESI $\dagger$ ). The \% of live/dead cells was achieved to support the evidence that the number of cells increases both at the surface and inside the structure, indicating that cells were able to adhere, migrate and proliferate inside the sponges.

AGS cell morphology in the MACE sponges was evaluated at different time points (Fig. 5), confirming the results obtained by the live-dead assay. AGS cells were able to organize in clusters and proliferate on the surface and core of the sponges (Fig. 5). AGS cells, when in 2D culture show a heterogeneous shape with a predominance of polygonal shape and the presence of very few elongated cells (Fig. S2A, ESI $\dagger$ ). In 3D culture, these cells are known to maintain a round shape and to aggregate into clusters. ${ }^{27}$ This type of behavior was recapitulated in MACE sponges (Fig. 5), probably due to the absence of

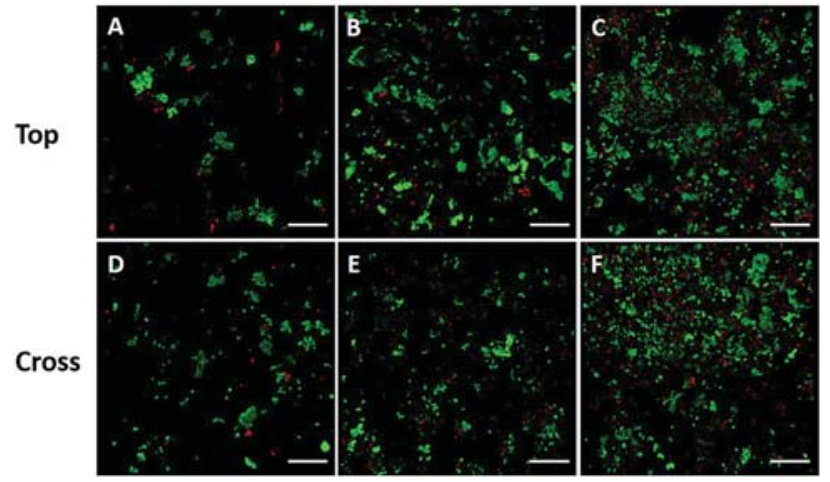

Fig. 4 Live-dead staining of AGS cells cultured in MACE sponges for 1 $(A$ and $D), 7$ ( $B$ and $E$ ) and 14 days ( $C$ and $F$ ). Cells are observed both at the surface $(A-C)$ and in the interior of the sponge (cross-sections; $D-F$ ). Bars correspond to $200 \mu \mathrm{m}$.

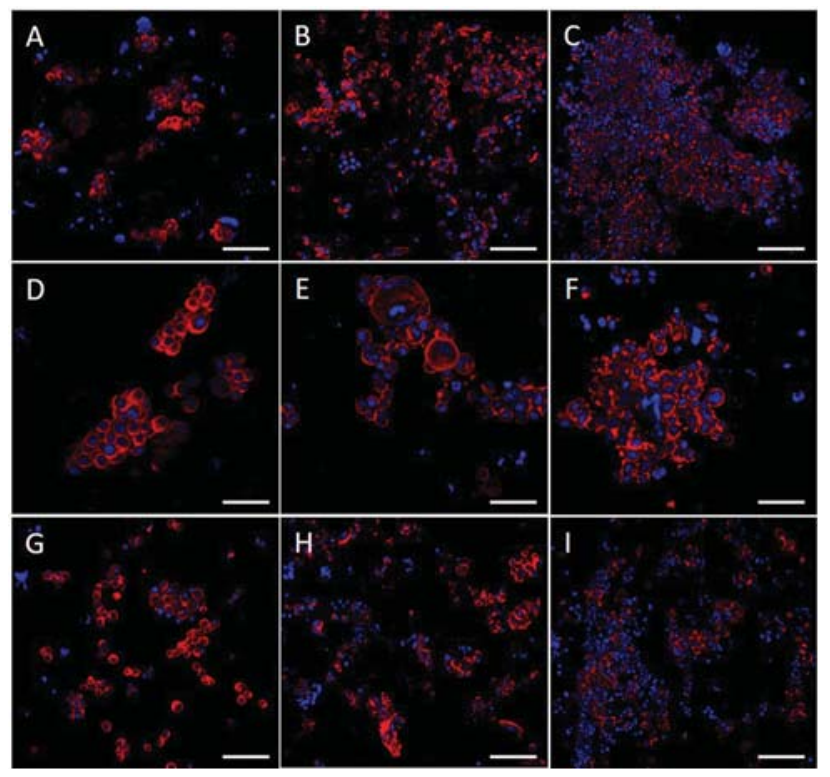

Fig. 5 Morphology of AGS cells cultured in MACE sponges for 1 (A, D and G), $7(B, E$ and $H)$ and 14 days (C, F and I). Cells are observed both at the surface $(A-F)$ and in the interior of the sponge (cross-sections; $G-I)$. Bars correspond to $100 \mu \mathrm{m}(\mathrm{A}-\mathrm{C}, \mathrm{G}-\mathrm{I})$ and $50 \mu \mathrm{m}(\mathrm{D}-\mathrm{F})$.

adhesion points. This same behavior was observed for ASCs cultured in MACE sponges (Fig. S2B, ESI $\dagger$ ). However, in the presence of fibronectin coating, ASCs were able to attach and showed the typical spindle shape (Fig. S2B, ESI $\dagger$ ).

Results obtained from DNA biochemical analysis show a clear proliferation tendency of AGS cells seeded onto MACE sponges, increasing up to 7 days $(p<0.001)$ (Fig. 6A). Metabolic activity and cell proliferation follow the same trend, increasing significantly up to 7 days $(p<0.001)$. The results obtained by dsDNA quantification suggest that the increase in metabolic activity observed with increasing time of culture can be attributed to cell proliferation (Fig. 6A and B). Considering the cell imaging (Fig. 5) and the proliferation data (Fig. 6), we can conclude that cells almost had no growth from day 7 to day 14. However, cells were not able to occupy the whole sponge indicating that either densely populated cells on the surface are causing limited nutrient transfer or it results from the low ratio of interconnected pores $(30.1 \pm 0.9 \%)$.
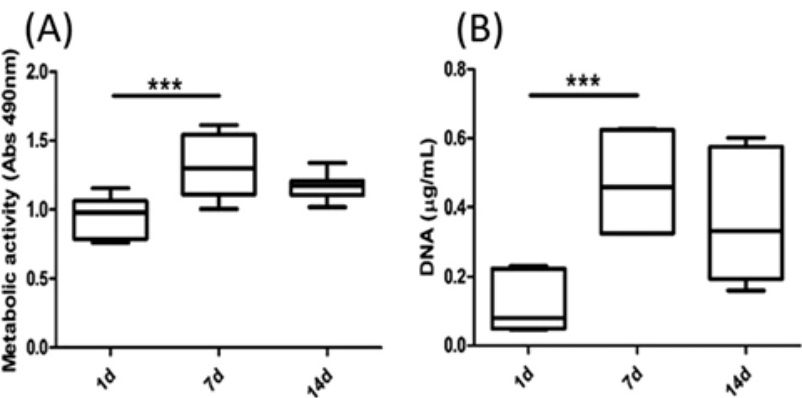

Fig. 6 Metabolic activity and DNA content of AGS cells cultured in MACE sponges. 


\section{Conclusions}

Modified acemannan containing unsaturated groups sensitive to UV irradiation was successfully prepared by reacting the acemannan with methacrylic anhydride. Furthermore, the photocrosslinking of the modified acemannan solution with UV led to the formation of acemannan sponges with uniform size, tuneable shape, and stability in an aqueous medium. Therefore, the developed photocrosslinked acemannan sponge present a useful performance that is not possible when acemannan powder is used. The photocrosslinked acemannan sponge was insoluble in water and showed good stability in PBS. Moreover, the developed 3D based structure can be biodegradable once implanted, during which the body could resorb it. On the other hand, the acemannan powder is soluble in water, which in turn could imply its rapid diffusion into the tissue in a short time that could be beneficial or not depending on the target tissue.

The biological behavior on photocrosslinked acemannan sponges revealed that they sustain gastric cancer cell viability during the time range tested. AGS cells were able to attach and spread on the surface and core of the sponges.

Further experiments will include longer culture times. The cellular response, together with the sponge's properties suggest that modified acemannan-based sponges could provide a suitable microenvironment system to further investigate cancer cell invasiveness and the effect of anti-cancer drugs. The present approach could significantly improve the processability of acemannan, allowing the production of high added-value structures, and enhance the biomedical value of this plant-derived polymer.

\section{Conflicts of interest}

The authors declare that there is no conflict of interest.

\section{Acknowledgements}

The authors Simone S Silva, and Diana Soares da Costa, acknowledge financial support from Portuguese Foundation for Science and Technology - FCT (SFRH/BPD/112140/2015, SFRH/ BPD/85790/2012, respectively). This work was also financially supported by the European Union Seventh Framework Programme (FP7/2007-2013) under grant agreement no. REGPOT-CT2012316331-POLARIS. The authors would like to express thanks to Lorand Laboratories (Texas, USA) for the Acemannan (BiAloeÒ) supply.

\section{References}

1 S. S. Silva, E. G. Popa, M. E. Gomes, M. Cerqueira, A. P. Marques, S. G. Caridade, P. Teixeira, C. Sousa, J. F. Mano and R. L. Reis, Acta Biomater., 2013, 9, 6790-6797.

2 M. Greenwell and P. Rahman, Int. J. Pharma Sci. Res., 2015, 6, 4103-4112.

3 M. Minamisawa, H. Minamisawa, S. Yoshida and N. Takai, Green Chem., 2005, 7, 595-601.
4 R. Mohammadinejad, S. Karimi, S. Iravani and R. S. Varma, Green Chem., 2016, 18, 20-52.

5 J. H. Hamman, Molecules, 2008, 13, 1599-1616.

6 M. Alonso, Y. Támbara, M. López, J. C. Aguilar, O. Mayo, E. Prieto, J. Cremata, G. Gerwig, H. Kamerling and E. Hardy, Biotecnol, 2012, 29, 87-101.

7 G. D. Sierra-Garcia, R. Castro-Rios, A. Gonzalez-Horta, J. LaraArias and A. Chavez-Montes, Nat. Prod. Commun., 2014, 9, 1217-1221.

8 A. Chauhan, S. Zubair, A. Sherwani and M. Owais, PLoS One, 2012, 7, e32049.

9 P. Sahu, D. Giri, R. Singh, P. Pandey, S. Gupta, A. Shrivastava, A. Kumar and K. Pandey, Pharmacol. Pharm., 2013, 4, 599-610.

10 S. Jettanacheawchankit, S. Sasithanasate, P. Sangvanich, W. Banlunara and P. Thunyakitpisal, J. Pharmacol. Sci., 2009, 109, 525-531.

11 P. Chantarawaratit, P. Sangvanich, W. Banlunara, K. Soontornvipart and P. Thunyakitpisal, J. Periodontal Res., 2014, 49, 164-178.

12 N. Jittapiromsak, D. Sahawat, W. Banlunara, P. Sangvanich and P. Thunyakitpisal, Tissue Eng., Part A, 2010, 16, 1997-2006.

13 S. Dusida, K. Suparaporn, S. Polkit, T. Takashi, K. Masae and T. Pasutha, J. Med. Plants Res., 2012, 6, 4069-4076.

14 S. Boonyagul, W. Banlunara, P. Sangvanich and P. Thunyakitpisal, Odontology, 2014, 102, 310-317.

15 B. Baroli, J. Chem. Technol. Biotechnol., 2006, 81, 491-499.

16 S. Mihaila, A. Gharwar, R. L. Reis, A. Marques, M. E. Gomes and K. Ali, Adv. Healthcare Mater., 2013, 2, 895-907.

17 A. Morelli and F. Chiellini, Macromol. Chem. Phys., 2010, 211, 821-832.

18 L. L. Reys, S. S. Silva, D. C. Soares, N. Oliveira, J. F. Mano, R. L. Reis and T. H. Silva, ACS Biomater. Sci. Eng., 2016, 2, 1151-1161.

19 J. Silva-Correia, J. M. Oliveira, S. G. Caridade, J. T. Oliveira, R. A. Sousa, J. F. Mano and R. L. Reis, J. Tissue Eng. Regener. Med., 2011, 5, e97-e107.

20 S. Talukdar, M. Mandal, D. W. Hutmacher, P. J. Russell, C. Soekmadji and S. C. Kundu, Biomaterials, 2011, 32, 2149-2159.

21 A. Mahmoudzadeh and H. Mohammadpour, J. Food Drug Anal., 2016, 24, 620-626.

22 F. M. Kievit, K. Wang, A. E. Erickson, S. K. Lan Levengood, R. G. Ellenbogen and M. Zhang, Biomater. Sci., 2016, 4, 610-613.

23 J. Li, Y. Zhou, W. Chen, Z. Yuan, B. You, Y. Liu, S. Yang, F. Li, C. Qu and X. Zhang, ACS Appl. Mater. Interfaces, 2018, 10, 36641-36651.

24 W. Asghar, R. El Assal, H. Shafiee, S. Pitteri, R. Paulmurugan and U. Demirci, Mater. Today, 2015, 18, 539-553.

25 W. Xing, W. Guo, C. H. Zou, T. T. Fu, X. Y. Li, M. Zhu, J. H. Qi, J. Song, C. H. Dong, Z. Li, Y. Xiao, P. S. Yuan, H. Huang and X. Xu, J. Dermatol. Sci., 2015, 79, 101-109.

26 D. F. Coutinho, S. V. Sant, H. Shin, J. T. Oliveira, M. E. Gomes, N. M. Neves, A. Khademhosseini and R. L. Reis, Biomaterials, 2010, 31, 7494-7502.

27 M. P. Quezada, C. Salinas, M. Gotteland and L. Cardemil, J. Agric. Food Chem., 2017, 65, 10029-10039. 